\title{
Transport relaxation time and length scales in turbulent suspensions
}

\author{
PHILIPPE CLAUDIN ${ }^{1} \dagger$, FRANÇOIS CHARRU \\ AND BRUNO ANDREOTTI ${ }^{1}$ \\ ${ }^{1}$ Laboratoire de Physique et Mécanique des Milieux Hétérogènes (PMMH), \\ UMR 7636 ESPCI-CNRS, Université Paris Diderot-Université Pierre et Marie Curie, \\ 10 rue Vauquelin, 75005 Paris, France \\ ${ }^{2}$ Institut de Mécanique des Fluides de Toulouse-CNRS, Université de Toulouse, \\ 31400 Toulouse, France
}

(Received 5 May 2010; revised 27 July 2010; accepted 5 November 2010; first published online 11 February 2011)

We show that in a turbulent flow transporting suspended sediment, the unsaturated sediment flux $q(x, t)$ can be described by a first-order relaxation equation. From a mode analysis of the advection-diffusion equation for the particle concentration, the relaxation length and time scales of the dominant mode are shown to be the deposition length $H U / V_{\text {fall }}$ and deposition time $H / V_{\text {fall }}$, where $H$ is the flow depth, $U$ the mean flow velocity and $V_{\text {fall }}$ the sediment settling velocity. This result is expected to be particularly relevant for the case of sediment transport in slowly varying flows, where the flux is never far from saturation. Predictions are shown to be in quantitative agreement with flume experiments, for both net erosion and net deposition situations.

Key words: sediment transport, suspensions

\section{Introduction}

Suspension is an important mode for the transport of sediments by fluid flows. It occurs when the falling velocity of the particles is smaller than the turbulent velocity fluctuations, so that particles can remain suspended for a long time, trapped by turbulent eddies, before they eventually fall back on the bed because of gravity. In nature, one observes suspension in large rivers, i.e. in their downstream part, where a large amount of fine particles has been collected from the catchment basin. Rivers that ordinarily present bed-load transport (the moving particles remain close to the bed) can also experience suspension (the particles are present over the whole flow depth) when the water discharge is unusually large, e.g. during flood events.

Vertical concentration profiles and overall sediment fluxes are among the major issues (see the pioneering works of Rouse 1936, Vanoni 1946 or van Rijn 1984b). From the point of view of hydraulic engineering, the problem has been satisfactorily solved for rivers in a steady state, although some questions are still open, such as particle trapping by turbulent eddies or the structure of the flow near the bottom where the concentration is large (Nielsen 1992; Nezu 2005). However, the response 
(a)

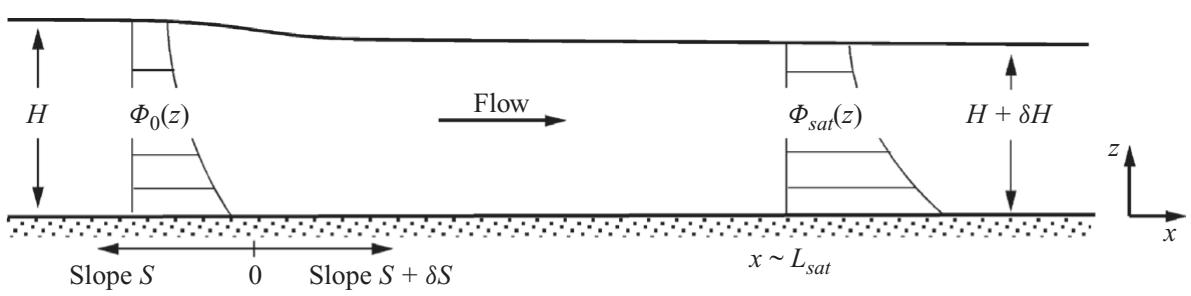

(b)

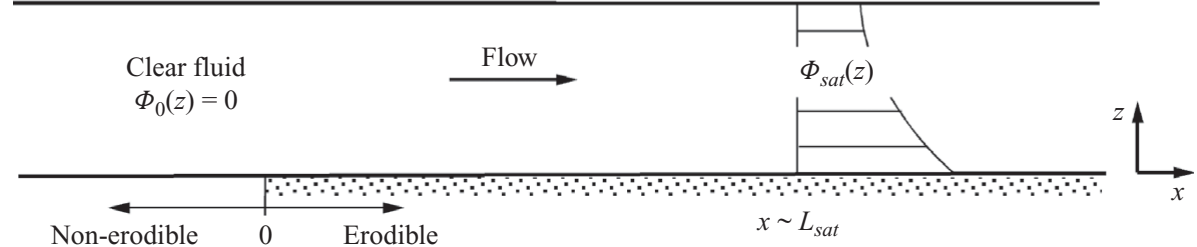

FIGURE 1. Sketches of the situations studied in $\S 4:(a)$ flow over a slope change and $(b)$ flow over the passage from non-erodible to erodible bed. Here, $H$ and $S$ are the river depth and the bottom slope for $x<0$, and $\Phi_{0}(z)$ is the corresponding saturated concentration profile; $\delta H$ and $\delta S$ are the variations in $H$ and $S$ downstream of the change at $x=0$, and $\Phi_{\text {sat }}(z)$ is the new saturated concentration profile reached at the distance $x \approx L_{\text {sat }}$.

of the sediment flux to temporal or spatial changes of the flow is largely unknown. Such changes may be induced, for instance, by long gravity waves, or a sudden increase of the flow rate, or variations of the river slope or geometry. Two typical problems of relaxation downstream of a change in the flow conditions are depicted in figure 1 , which will be studied in $\S 4$ : that of a small change of the slope of the bottom (figure $1 a$ ) and that of a change of the bottom conditions, from non-erodible to erodible (figure $1 b$ ). The suspended sediment response is expected to have a strong effect on the dynamics of the erodible bottom, especially on the formation of dunes or bars or, at a larger scale, on the development of meanders (Seminara 2006). Specific relaxation problems have been investigated by numerical integration of the Reynolds-averaged Navier-Stokes equations, using mixing length or $k-\varepsilon$ turbulence models (Apmann \& Rumer 1970; Hjelmfelt \& Lenau 1970; Jobson \& Sayre 1970; van Rijn 1986b; Celik \& Rodi 1988; Ouillon \& Le Guennec 1996).

In the case in which bed load is dominant, or in the aeolian situation (saltation), it has been shown that the evolution of the sediment flux $q$ can be accounted for by a relaxation equation of the form

$$
T_{\text {sat }} \partial_{t} q+L_{\text {sat }} \partial_{x} q=q_{\text {sat }}-q,
$$

where $q_{\text {sat }}$ is the saturated flux; $T_{\text {sat }}$ and $L_{\text {sat }}$ are the relaxation time and length scales i.e. the time and length over which the flux relaxes towards saturation. This saturation corresponds to the homogeneous and steady state for which sediment transport is constant in both space and time for given flow conditions. Such a first-order equation was first introduced in the aeolian context as the simplest equation describing relaxation effects (Sauermann, Kroy \& Herrmann 2001; Andreotti, Claudin \& Douady 2002; Kroy, Sauermann \& Herrmann 2002; Andreotti 2004). It was then shown that, for water flows, this equation can be derived from the analysis of the erosion and deposition rates, the relaxation scales there being related to particle deposition (and not particle inertia) (Charru 2006; Lajeunesse, Malverti \& Charru 2010). The importance of the relaxation length $L_{s a t}$ appeared to be crucial in particular for 
stability analyses of the erodible bottom, for selection of the ripple wavelength (Fourrière, Claudin \& Andreotti 2010). The importance of relaxation phenomena for suspensions in turbulent flow is well known in the context of hydraulic engineering (Yalin \& Finlaysen 1973; van Rijn 1986a; Celik \& Rodi 1988; Ouillon \& Le Guennec 1996). This importance has also been recognized in the context of geomorphology; in particular, Davy \& Lague (2009) proposed a deposition length of sediment as the relevant transport length. However, a derivation of a relaxation equation of the form (1.1), from firm hydrodynamic grounds, is still lacking.

In this paper, we discuss the conditions under which an equation of the form (1.1) can be derived for turbulent flows, when suspension is the dominant mode of transport, with particular emphasis on the identification of the saturation length and time scales. The paper is organized as follows. In the next section, we present the flow models and saturation conditions. In $\S 3$ we perform a mode analysis of the advection-diffusion equation for the particle concentration applied to unsaturated cases and then identify the saturation length and time scales of the flux. The relevance of this approach is illustrated in $\S 4$ by treating a few examples: (i) the effect on the sediment flux of a change in the river slope, (ii) the change from a fixed to an erodible bed and (iii) the deposition of sediments from a source near the free surface. For the last two situations, the predictions of the model are tested against experimental data from the literature.

\section{Flow models}

\subsection{Logarithmic flow model}

We consider the free-surface, turbulent flow of a fluid layer of thickness $H$ over an erodible bed. For the sake of simplicity, we restrict the discussion to flows invariant in the spanwise direction, i.e. two-dimensional, with streamwise coordinate $x$ and upward transverse coordinate $z$. Measurements have shown that the profile of the streamwise velocity is close to the logarithmic law,

$$
u_{x}(z)=\frac{u_{*}}{\kappa} \ln \left(\frac{z+z_{0}}{z_{0}}\right),
$$

where $u_{*}$ is the friction velocity; $z_{0}$ is the hydrodynamical bed roughness; and $\kappa=0.4$ is the von Kármán coefficient. For a steady flow in which the shear stress is balanced by the streamwise component of gravity, the shear stress increases linearly from zero at the free surface to $\tau_{b}=\rho u_{*}^{2}$ at the bottom, so that the logarithmic velocity profile (2.1) corresponds to a parabolic eddy viscosity $v_{t}$ (Nezu \& Rodi 1986), given by

$$
\frac{v_{t}}{u_{*} H}=\kappa\left(\frac{z+z_{0}}{H}\right)\left(1-\frac{z}{H}\right) \text {. }
$$

From (2.1), the depth-averaged velocity $U$ is given by

$$
\lambda \equiv \frac{U}{u_{*}}=\frac{1}{\kappa}\left[\ln \left(\frac{H}{z_{0}}\right)-1\right],
$$

with the typical value $\lambda=10$, corresponding to $z_{0} / H \approx 0.01$ (Raudkivi 1998).

We assume that the sediment concentration $\phi$ is governed by the advection-diffusion equation

$$
\frac{\partial \phi}{\partial t}+u_{x} \frac{\partial \phi}{\partial x}=\frac{\partial}{\partial x}\left(D \frac{\partial \phi}{\partial x}\right)+\frac{\partial}{\partial z}\left(D \frac{\partial \phi}{\partial z}+\phi V_{\text {fall }}\right),
$$


where $D$ is the particle eddy diffusivity and $V_{\text {fall }}$ the settling velocity. Measurements have shown that $D(z)$ is reasonably parabolic and proportional to the eddy viscosity (2.2), with a turbulent Schmidt number

$$
S c=\frac{v_{t}}{D}
$$

in the range 0.5-1 (Coleman 1970; Celik \& Rodi 1988; Nielsen 1992). The settling velocity $V_{\text {fall }}$ is taken uniform and, when needed for comparison with experiments, equal to that of a single particle in the quiescent fluid. Note that the above modelling ignores inertial effects on particle motion, in particular their ejection from the core of vortices and their clustering (Bec et al. 2007; Hunt et al. 2007). We also limit the discussion to dilute suspensions, i.e. small volume particle concentration $\phi$, for which there is no significant feedback of the particles on transport.

Solving (2.4) requires two boundary conditions, one at the free surface and one on the sedimentary bed. At the free surface, the net vertical flux vanishes, giving

$$
D \frac{\partial \phi}{\partial z}+\phi V_{\text {fall }}=0 \quad \text { at } \quad z=H .
$$

At the bottom, just above the bed-load layer where particles mainly roll and slide on each other, the diffusive flux is equal to the erosion flux $\varphi_{\uparrow}$, i.e. the volume of particles entrained in suspension per unit time and bed area (Parker 1978; van Rijn 1986b):

$$
-D \frac{\partial \phi}{\partial z}=\varphi_{\uparrow} \quad \text { at } \quad z=0
$$

The erosion rate, or 'pickup function', is generically an increasing function of the basal shear stress above a threshold. Its functional form is determined phenomenologically from experiments and depends on the nature of the bed whether it is consolidated/cohesive or not, composed of grains or containing clay, etc. (Shields 1936; Einstein 1950; Engelund 1970; van Rijn 1984a; Briaud et al. 2001; Hanson \& Simon 2001; Bonelli, Brivois \& Benahmed 2007).

Two remarks have to be made here. First, the bottom condition (2.7) applies for steady and homogeneous as well as unsteady and heterogeneous flows. In the latter case, the erosion flux may be different from the deposition flux, so that the net flux is non-zero, which may lead to variations of the bed topography (but not necessarily, as in the experiments to be discussed later). Possible variations in the bed topography will be ignored here. Second, the boundary condition (2.7) corresponds to a bed allowing unlimited sediment supply. For more general situations (e.g. fixed bed), slightly different boundary conditions have been proposed (see Celik \& Rodi 1988), which however requires an empirical constant or reference concentration near the bottom to be given, which varies along the channel. Finally, assuming that particles have the same mean velocity as the fluid, $u_{x}$, the flux of suspended particles, per unit length in the spanwise direction, is given by

$$
q=\int_{0}^{H} \phi u_{x} \mathrm{~d} z
$$

The concentration equation (2.4) with the boundary condition (2.6) admits a steady and homogeneous solution corresponding to the balance of the settling and diffusive fluxes,

$$
\Phi_{\text {sat }}(z)=\Phi_{b}\left(\frac{1-z / H}{1+z / z_{0}}\right)^{\beta /\left(1+z_{0} / H\right)}
$$



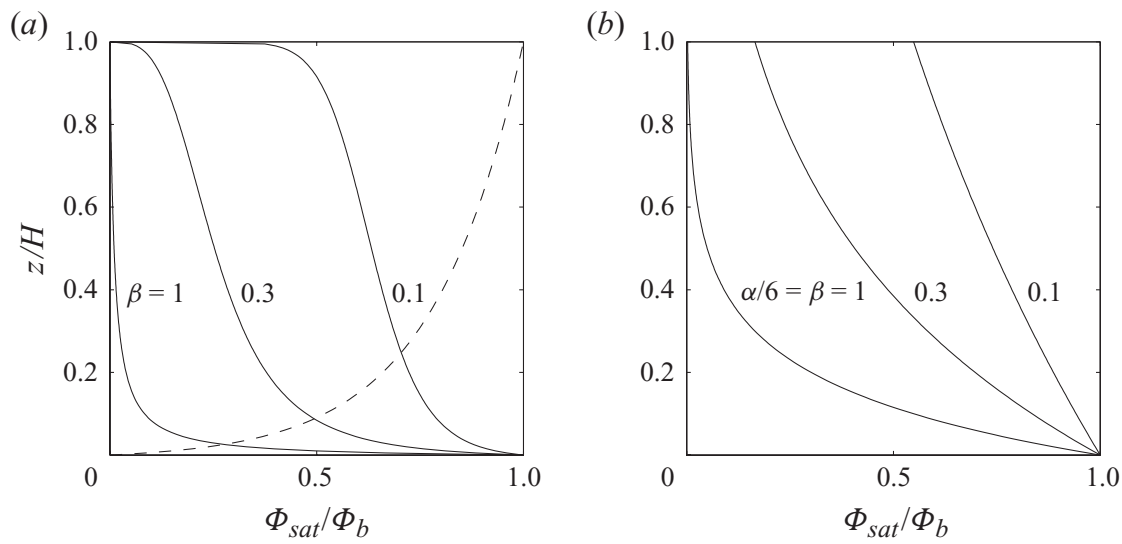

FiguRE 2. (a) Logarithmic flow model: normalized velocity profile (dashed line) and normalized concentration profiles (2.9) for three values of $\beta$ (solid lines). (b) Plug flow model: normalized concentration profiles (2.12) for the corresponding values of $\alpha=6 \beta$.

where $\beta$, known as the Rouse number, is defined as $\beta=\left(S c V_{\text {fall }}\right) /\left(\kappa u_{*}\right)$, and the bottom concentration $\Phi_{b}=\Phi_{\text {sat }}(0)$ is determined from the condition (2.7) as

$$
\Phi_{b}=\frac{\varphi_{\uparrow}\left(\tau_{b}\right)}{V_{\text {fall }}} .
$$

Note that (2.9) differs slightly for the classical expression of the Rouse profile (Nielsen 1992) because the location at which the velocity (2.1) vanishes and the bottom boundary condition (2.7) applies has been chosen to be $z=0$ instead of $z=z_{0}$. Suspension typically occurs when $V_{\text {fall }}<0.8 u_{*}$ (Fredsøe \& Daigaard 1992), which corresponds to $\beta<4 / 3$ with $S c=2 / 3$. Figure 2(a) displays the velocity profile (2.1), normalized by $u_{x}(H)$, and the concentration profile (2.9), normalized by $\Phi_{b}$, for three typical values of $\beta$.

\subsection{A simplified plug flow model}

In order to get analytical results, a simplified plug flow model will be used in the following, which appears to provide accurate results as long as the assessment of the relaxation equation (1.1) is pursued. This model corresponds to the uniform flow velocity $u_{x}(z)=U$ and the friction velocity $u_{*}=U / \lambda$, where $\lambda$ is the same constant as in the previous section. Such a plug flow model is of course a rough description, and the velocity profile actually does not switch from zero on the bed to its average value on a vanishing vertical distance, leading to an infinite shear. This problem is not present in the logarithmic model, which is more realistic from this point of view. However, as shown below, these two models do not differ much as far as the relaxation modes are concerned, which means that what occurs very close to the bed is not very important for the present purpose. Accordingly, a uniform particle diffusivity $D_{0}$ will be taken in the concentration equation (2.4) and boundary conditions (2.6) and (2.7), equal to the average of the parabolic distribution given by (2.2) and (2.5). Up to a small correction of order $z_{0} / H$, the diffusivity $D_{0}$ is given by

$$
\frac{D_{0}}{u_{*} H}=\frac{\kappa}{6 S c} \equiv \mathscr{K} \text {. }
$$


With this uniform diffusivity $D_{0}$, the advection-diffusion equation (2.4) admits the steady and homogeneous solution

$$
\Phi_{\text {sat }}=\Phi_{b} \exp \left(-\alpha \frac{z}{H}\right) \quad \text { with } \quad \alpha \equiv \frac{V_{\text {fall }} H}{D_{0}}=6 \beta,
$$

which also satisfies the boundary condition (2.6) at the free surface. The boundary condition at the bed (2.7) determines the bed concentration (2.10). Figure 2(b) displays the concentration profile (2.12), normalized by $\Phi_{b}$, for three typical values of $\alpha=6 \beta$. It can be seen that for the same value of the Rouse number, the plug flow model predicts a sediment concentration slightly larger than that of the logarithmic flow model. Small values of $\alpha$ correspond to strong suspensions, i.e. situations for which the sediment is distributed almost uniformly over the whole depth of the flow. This is achieved when the settling velocity is small (very fine particles) or when the diffusivity is large (large flow velocity). Finally, the saturated particle flux per unit width, $q_{\text {sat }}$, normalized by the water flux $U H$, is given by

$$
\frac{q_{\text {sat }}}{U H}=\frac{1}{U H} \int_{0}^{H} \Phi_{\text {sat }} U \mathrm{~d} z=\frac{1-\mathrm{e}^{-\alpha}}{\alpha} \Phi_{b} .
$$

For small $\alpha$, this dimensionless flux tends to $\Phi_{b}$, as expected.

\section{Non-homogeneous and unsteady flows}

In this section, we successively consider a spatial and a temporal evolution problem (§§3.1 and 3.2 respectively). These problems are solved using a mode analysis; i.e. the departure of the concentration field from the saturated distribution $\Phi_{\text {sat }}(z)$ is decomposed as a sum of terms of the form $c(x, t) f(z)$. It is shown that, for the spatial problem, there exists a discrete set of amplitudes $c_{n}(x) \propto \mathrm{e}^{-x / L_{n}}$, and for the temporal problem, there exists a similar set of amplitudes $c_{n}(t) \propto \mathrm{e}^{-t / T_{n}}$. Then the sediment flux is shown to be dominated by the mode with the largest length or time, $L_{1}$ or $T_{1}$. This result demonstrates that for large-scale problems, the relaxation equation (1.1) retains the most important features of unsaturated sediment transport, with relaxation scales $L_{\text {sat }}$ and $T_{\text {sat }}$ equal to the largest scales arising from the mode analysis.

The analytical calculations presented below use the plug flow model because of its simplicity, in the spirit of the work of Mei (1969). Calculations for the logarithmic model are not reported in detail, but the corresponding results are plotted for comparison in some of the figures.

\subsection{Spatial evolution and the relaxation lengths}

We consider the situation in which, for the given flow conditions and the corresponding saturated concentration profile $\Phi_{\text {sat }}(z)$, the actual concentration profile at some point, say $x=0$, is $\Phi_{\text {sat }}(z)-\phi(x=0, z)$, where $\phi(x, z)$ is a 'concentration defect'. We search for the distance at which the saturated distribution $\Phi_{\text {sat }}(z)$ is recovered, corresponding to vanishing $\phi(x, z)$. Looking for normal modes of relaxation of the concentration defect of the form

$$
\phi(x, z)=\Phi_{b} f(z) \exp (-x / L)
$$

we get from the advection-diffusion equation (2.4)

$$
\left(\frac{\lambda u_{*}}{L}+\frac{D}{L^{2}}\right) f+\frac{\mathrm{d}}{\mathrm{d} z}\left(D \frac{\mathrm{d} f}{\mathrm{~d} z}+f V_{\text {fall }}\right)=0 .
$$


(a)

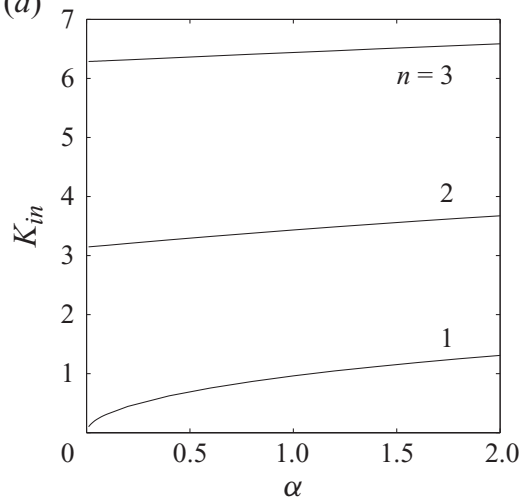

(b)

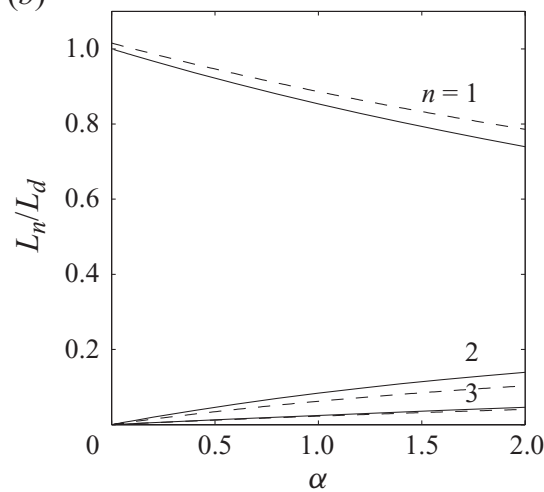

FiguRE 3. (a) Variation with $\alpha$ of the three smallest roots of (3.6). (b) Corresponding relaxation normalized lengths $L_{n} / L_{d}$ for $\lambda / \mathscr{K}=50$ : solid lines, plug flow; dashed lines, logarithmic flow.

At the free surface, the zero flux condition (2.6) gives

$$
D \frac{\mathrm{d} f}{\mathrm{~d} z}+f V_{\text {fall }}=0 \quad \text { at } \quad z=H .
$$

On the bed the friction velocity $u_{*}$ is assumed to be uniform. The erosion flux $\varphi_{\uparrow}$, which depends only on $u_{*}$, is uniform too. Hence, the disturbance of $\varphi_{\uparrow}$ is zero, so that, from (2.7),

$$
\frac{\mathrm{d} f}{\mathrm{~d} z}=0 \quad \text { at } \quad z=0 .
$$

The above differential problem is solved numerically for parabolic $D$ (logarithmic flow model) and analytically for uniform $D=D_{0}$ (plug flow model). For uniform $D_{0}$, (3.2) has solutions of the form $f(z) \propto \exp (K z / H)$, where $K$ has to satisfy a q uadratic equation with roots $K_{+}$and $K_{-}$given by

$$
K_{ \pm}=-\frac{\alpha}{2} \pm \mathrm{i} K_{i} \quad \text { with } \quad K_{i}=\sqrt{\frac{\lambda}{\mathscr{K}} \frac{H}{L}+\frac{H^{2}}{L^{2}}-\frac{\alpha^{2}}{4}} .
$$

Then the boundary conditions (3.3) and (3.4) select a discrete set of relaxation lengths $L$, satisfying

$$
\tan K_{i}=\left(\frac{K_{i}}{\alpha}-\frac{\alpha}{4 K_{i}}\right)^{-1} .
$$

This equation has an infinite number of real positive solutions $K_{i n}, n \geqslant 1$. Figure $3(a)$ shows the variation with $\alpha$ of the three smallest ones $(n=1,2,3)$. For small $\alpha$, these solutions behave as $K_{i 1} \sim \sqrt{\alpha}$ and $K_{i n} \sim(n-1) \pi$ for $n \geqslant 2$.

The corresponding relaxation lengths $L_{n}$ are found from (3.5):

$$
\frac{H}{L_{n}}=\frac{1}{2}\left(-\frac{\lambda}{\mathscr{K}} \pm \sqrt{\left(\frac{\lambda}{\mathscr{K}}\right)^{2}+\alpha^{2}+4 K_{\text {in }}^{2}}\right), \quad n \geqslant 1 .
$$

They are displayed for $n=1,2,3$ in figure $3(b)$ as a function of $\alpha$ (solid lines), normalized with the characteristic deposition length

$$
L_{d} \equiv \frac{U}{V_{\text {fall }}} H \text {. }
$$



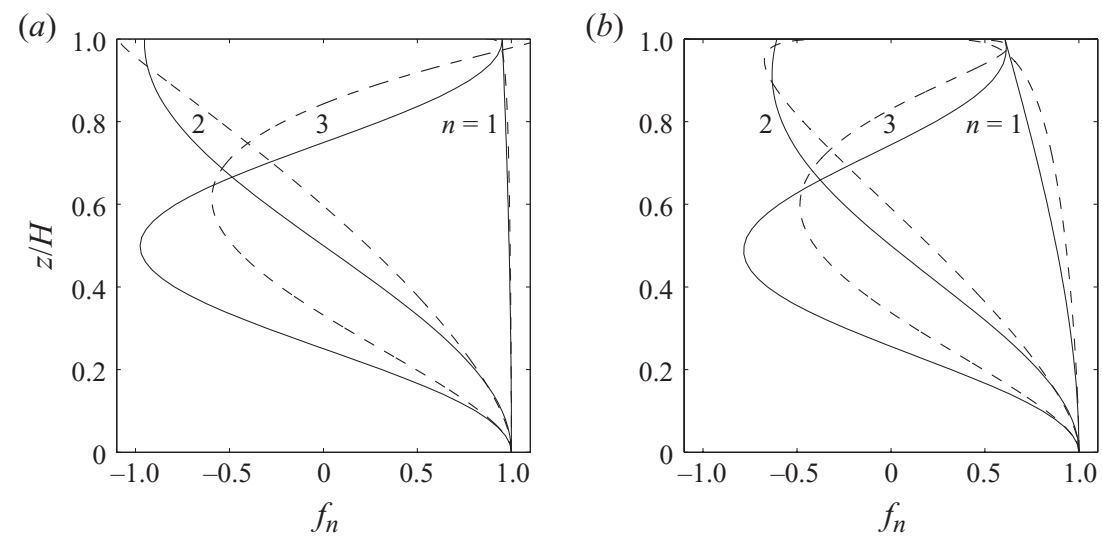

Figure 4. Profile of the three first eigenfunctions $f_{n}(z)$, for $\alpha=(a) 0.1$ and $(b) 1$ : solid lines, plug flow; dashed lines, logarithmic flow.

It can be seen that $L_{1}$ is much larger than the higher-order relaxation lengths typically by one order of magnitude. Remarkably, in the limit of small $\alpha$ (large flow velocity or small settling velocity), the largest length $L_{1}$ tends to $L_{d}$, whereas higher-order lengths remain of the order of the flow depth $H$ :

$$
L_{1} \sim L_{d}, \quad L_{n} \sim \frac{\lambda / \mathscr{K}}{(n-1)^{2} \pi^{2}} H \quad \text { for } n \geqslant 2 .
$$

Figure 3(b) also displays the normalized relaxation lengths obtained from the logarithmic flow model (dashed lines), from numerical integration of (3.2) with parabolic $D$. It can be seen that these lengths are close to those from the plug flow model, especially for the largest length $L_{1}$. Note that $L_{n} / L_{d}$ is weakly sensitive to the value of $\lambda / \mathscr{K}$ : doubling this ratio does not bring any visible change, at least for $\alpha \leqslant 2$.

The eigenfunctions $f_{n}(z)$ are given by

$$
f_{n}(z)=\left[\cos \left(K_{i n} \frac{z}{H}\right)+\frac{\alpha}{2 K_{i n}} \sin \left(K_{i n} \frac{z}{H}\right)\right] \exp \left(-\frac{\alpha}{2} \frac{z}{H}\right), \quad n \geqslant 1,
$$

with the normalization condition $f_{n}(0)=1$. These eigenfunctions are displayed in figure 4 for $n=1,2,3$ (solid lines), for $\alpha=0.1$ (figure $4 a$ ) and $\alpha=1$ (figure $4 b$ ). It can be seen that $f_{1}(z)$ decreases slightly and monotonically from the bottom to the top, whereas higher-order eigenfunctions oscillate, more and more strongly with increasing $n$. Figure 4 also displays the eigenfunctions from the logarithmic flow model (dashed lines). It can be seen that for the mode associated with the largest length $L_{1}(n=1)$, eigenfunctions of both models remain very close to each other and that differences become larger as $n$ increases.

Let us turn to the sediment flux. The contribution of the $n$th eigenmode to the sediment flux $Q_{n}$, normalized with the characteristic sediment flux $U H \Phi_{b}$ and the exponential $x$-dependence, is

$$
\frac{1}{\exp \left(-x / L_{n}\right)} \frac{1}{\Phi_{b} U H} Q_{n}=\frac{1}{H} \int_{0}^{H} f_{n}(z) \mathrm{d} z .
$$

Table 1(a) displays the contribution of each of the first three modes to the sediment flux, i.e. the right-hand side of the above equation. It can be seen that the contribution 


\begin{tabular}{|c|c|c|c|c|}
\hline & \multicolumn{3}{|c|}{ (a) } & \\
\hline$\alpha$ & 1 & 2 & 3 & \\
\hline 0.1 & 0.9836 & 0.0099 & 0.0025 & \\
\hline 1 & 0.8533 & 0.0832 & 0.0240 & \\
\hline & & (b) & & \\
\hline$\alpha$ & $A_{1}$ & $A_{2}$ & $A_{3}$ & $A_{4}$ \\
\hline 0.1 & 0.967 & 0.020 & 0.005 & 0.002 \\
\hline 1 & 0.724 & 0.150 & 0.047 & 0.022 \\
\hline
\end{tabular}

TABLE 1. (a) Contribution of the three lowest-order eigenmodes to the normalized sediment flux (right-hand side of (3.11)), for $\alpha=0.1$ and 1. (b) Normalized coefficients $A_{n}=a_{n} / \delta \Phi_{b}$ of the expansion (4.2), computed from a projection over four modes.

of the first mode $n=1$ strongly dominates. The smallness of the contribution of the higher-order modes is due to the oscillations of the eigenfunctions, as shown in figure 4. For small $\alpha$, the normalized flux is close to 1 for $n=1$ and decreases as $\alpha /((n-1) \pi)^{2}$ for $n \geqslant 2$.

The general form of the concentration defect finally is

$$
\phi(x, z)=\Phi_{b} \sum_{n=1}^{\infty} a_{n} f_{n}(z) \exp \left(-x / L_{n}\right),
$$

where the relaxation lengths $L_{n}$ are given by (3.6) and (3.7); the eigenfunctions $f_{n}(z)$ are given by (3.10); and the coefficients $a_{n}$ have to be determined by the concentration profile imposed at $x=0$. Such a determination will be illustrated in $\S 4$.

\subsection{Temporal evolution and relaxation times}

We now consider an unsaturated concentration profile at initial time $t=0$, say $\Phi_{\text {sat }}(z)-\phi(z, t=0)$, uniform in the streamwise $x$-direction, and search for the time needed for relaxation to the saturated distribution $\Phi_{\text {sat }}(z)$ given by (2.12), i.e. vanishing concentration defect $\phi(z, t)$. Calculations go along the same lines as in the previous subsection, so they are only briefly sketched here. Looking for normal modes of the form

$$
\phi(t, z)=\Phi_{b} g(z) \exp (-t / T),
$$

we get from (2.4) the equation governing the eigenfunctions $g(z)$ :

$$
\frac{1}{T} g+\frac{\mathrm{d}}{\mathrm{d} z}\left(D_{0} \frac{\mathrm{d} g}{\mathrm{~d} z}+g V_{\text {fall }}\right)=0 .
$$

This equation has solutions of the form $g(z) \propto \exp (K z / H)$, where $K$ has to satisfy a quadratic equation with roots $K_{+}$and $K_{-}$defined as

$$
K_{ \pm}=-\frac{\alpha}{2} \pm \mathrm{i} K_{i} \quad \text { with } \quad K_{i}=\sqrt{\frac{H}{\mathscr{K} u * T}-\frac{\alpha^{2}}{4}} .
$$

The boundary conditions at $z=0$ and $z=H$ are the same as in the previous section, so that $K_{i}$ still satisfies (3.6), with same solutions $K_{i n}, n \geqslant 1$. The corresponding relaxation times $T_{n}$ are then given by

$$
\frac{H}{\mathscr{K} u_{*} T_{n}}=K_{\text {in }}^{2}+\frac{\alpha^{2}}{4}, \quad n \geqslant 1 .
$$


Introducing the characteristic deposition time

$$
T_{d} \equiv \frac{H}{V_{\text {fall }}}=\frac{L_{d}}{U}
$$

the relaxation times are, in the limit of small $\alpha$,

$$
T_{1} \sim T_{d}, \quad T_{n} \sim \frac{\alpha T_{d}}{(n-1)^{2} \pi^{2}} \sim \frac{L_{n}}{U} \quad \text { for } n \geqslant 2 .
$$

As for the spatial problem, the sediment dynamics is dominated by the largest time $T_{1}$, equal to the deposition time $T_{d}$ for strong suspensions.

\section{Two illustrations, and comparison with experiments}

\subsection{Effect of a change in the bed slope}

Consider the situation depicted in figure $1(a)$, of a flow with the saturated concentration profile $\Phi_{0}(z)$ which experiences a small variation $\delta S$ in the bottom slope at $x=0$, either positive or negative. This variation leads to a small change of the water depth and friction velocity, according to $\delta H / H=-\delta u_{*} / u_{*}=-(1 / 2) \delta S / S$. This change occurs on a hydrodynamic length scale $L_{h}$ given by the balance between the acceleration $U \delta U / L_{h}$ and the force $g \delta S$, i.e. $L_{h} / L_{d}=U V_{\text {fall }} / 2 u_{*}^{2}=\lambda \mathscr{K} \alpha / 2$. The present analysis is valid for small $L_{h} / L_{d}$, a condition which is fulfilled for small $\alpha$.

The change in the saturated concentration profile due to the slope variation $\delta S$ is, at the linear order in $\delta u_{*}$,

$$
\delta \Phi(z)=\delta \Phi_{b} \exp \left(-\alpha \frac{z}{H}\right) \quad \text { with } \quad \delta \Phi_{b}=\frac{\varphi_{\uparrow}^{\prime}\left(u_{*}\right) \delta u_{*}}{V_{\text {fall }}},
$$

where $\varphi_{\uparrow}^{\prime}\left(u_{*}\right)$ is the derivative of the erosion rate $\varphi_{\uparrow}\left(u_{*}\right)$. The concentration defect at $x=0$ corresponds to this change $(\phi(0, z)=\delta \Phi(z))$, so that the coefficients $a_{n}$ of the expansion (3.12) must satisfy

$$
\delta \Phi_{b} \exp \left(-\frac{\alpha}{2} \frac{z}{H}\right)=\sum_{n=1}^{\infty} a_{n}\left[\cos \left(K_{\text {in }} \frac{z}{H}\right)+\frac{\alpha}{2 K_{\text {in }}} \sin \left(K_{\text {in }} \frac{z}{H}\right)\right] .
$$

These coefficients can be determined from the projection of the above equation on the eigenfunctions, i.e. truncation of the sum on the right-hand side to $p$ terms, multiplication by each eigenfunction and integration of both sides from 0 to $H$. A linear system of $p$ equations is obtained, whose solution gives the coefficients $a_{1}, \ldots, a_{p}$. Table $1(b)$ displays the normalized coefficients $A_{n}=a_{n} / \delta \Phi_{b}$ resulting from the projection over $p=4$ modes, for two values of $\alpha$. One can see that the first mode captures most of the weight; the contribution of the second one is smaller but still significant, and higher modes are negligible. We have checked that considering more terms in the expansion has negligible effect on the dominant coefficients. The small weight of the oscillating modes is consistent with the slow variation with $z$ of the initial concentration profile (4.1).

Figure 5 displays profiles of the concentration defect $\phi(x, z)$ at the location of the slope change, $x / L_{d}=0$, and three positions downstream, for $\alpha=0.1$ (figure $5 a$ ) and $\alpha=1$ (figure $5 b$ ). It can be seen that at the position $x / L_{d}=3$, the concentration defect is nearly zero. The flux defect, i.e. the depth-integrated profiles of the concentration defect, correspondingly decays towards zero (not shown) and does so almost exponentially with relaxation length $L_{d}$, as predicted by (1.1). This confirms 


\begin{tabular}{|c|c|c|c|c|}
\hline & van Rijn (1986a) & \multicolumn{2}{|c|}{ Ashida \& Okabe (1982) } & Jobson \& Sayre \\
\hline & & Run 5 & Run 6 & Runs FS1 and \\
\hline & Erosion & Erosion & Deposition & Depositic \\
\hline Symbol & $*$ & 0 & $\square$ & $\mathbf{\square}$ \\
\hline$H(\mathrm{~cm})$ & 25 & \multicolumn{2}{|c|}{4.3} & 40.7 \\
\hline$U\left(\mathrm{~cm} \mathrm{~s}^{-1}\right)$ & 67 & \multicolumn{2}{|c|}{37.3} & 29.1 \\
\hline$u_{*}\left(\mathrm{~cm} \mathrm{~s}^{-1}\right)$ & 4.77 & \multicolumn{2}{|c|}{3.63} & 4.48 \\
\hline$V_{\text {fall }}\left(\mathrm{cm} \mathrm{s}^{-1}\right)$ & 2.2 & \multicolumn{2}{|c|}{1.85} & $1.0-2.0$ \\
\hline$L_{d}(\mathrm{~m})$ & 7.6 & \multicolumn{2}{|c|}{0.87} & $5.9-11.9$ \\
\hline$\alpha$ & - & 3.1 & 2.5 & 1.2 \\
\hline$S c$ & - & 0.41 & 0.33 & $0.18-0.36$ \\
\hline
\end{tabular}

TABLE 2. Hydraulic parameters $H, U, u_{*}$ and $V_{\text {fall }}$ of the experiments, $L_{d}=\left(U / V_{\text {fall }}\right) H, \alpha$ from the exponential fit of the downstream concentration profile and $S c=\kappa \alpha u_{*} / 6 V_{\text {fall }}$.
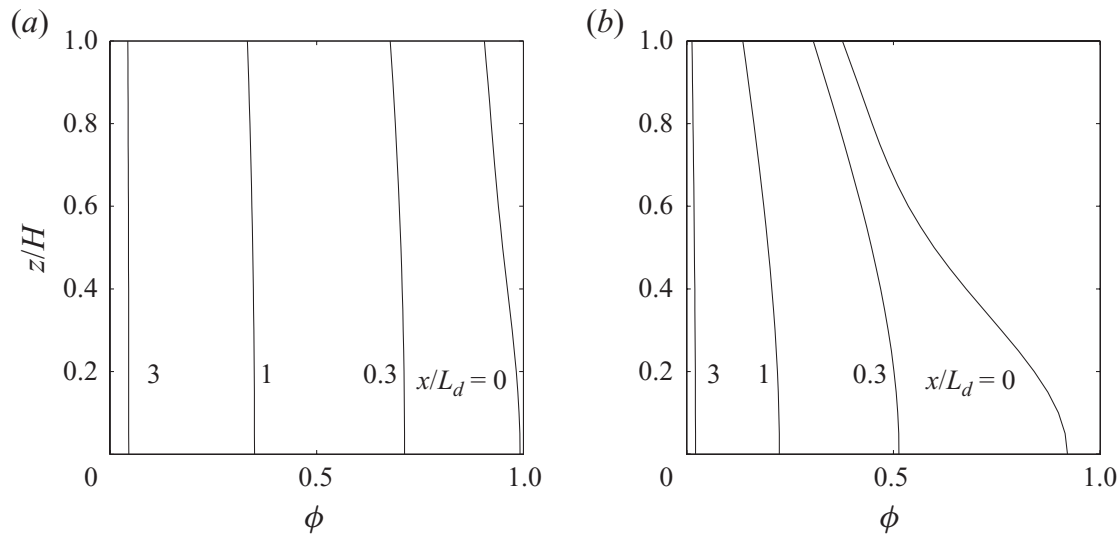

FIGURE 5. Relaxation of the concentration defect $\phi(x, z)$ after a slope change as sketched in figure 1 $(a)$, with summation over the three first modes (plug flow model). Profiles of $\phi(x, z)$ at the four downstream positions $x / L_{d}=0,0.3,1$ and 3, for $\alpha=(a) 0.1$ and $(b) 1$.

that the dominant mode with the relaxation length $L_{d}$ captures most of the sediment flux variations. For this example, as well as for the next ones, the question of the plug flow limit is not crucial: as far as the relaxation modes are concerned, the logarithmic and plug models do not differ much (figure 4).

\subsection{Net erosion experiments}

Another situation of interest is that of a flow of clear fluid on a non-erodible bed $\left(\Phi_{0}(z)=0\right)$ reaching an erodible bed lying in $x>0$, as sketched in figure $1(b)$. Suspension develops downstream until the saturated concentration profile $\Phi_{\text {sat }}(z)$ is reached. The analysis goes along the same lines as for the slope change. The concentration defect $\phi(x, z)$, can be decomposed on the eigenfunctions (3.10), with the coefficients $a_{n}$ determined by the concentration profile at $x=0$. The equation to be satisfied turns out to be the same as (4.2) with $\Phi_{b}$ instead of $\delta \Phi_{b}$. Thus the coefficients are $a_{n}=A_{n} \delta \Phi_{b}$ with the normalized coefficients $A_{n}$ given in table $1(b)$.

The prediction that the eigenmode with the largest relaxation length captures most of the sediment flux can be assessed from the experimental observations of van Rijn (1986a) and Ashida \& Okabe (1982) - non-Japanese readers can access these latter data from the paper of Celik \& Rodi (1988). These experiments precisely correspond to the sketch depicted in figure $1(b)$. Their hydraulic parameters are given in table 2 . 

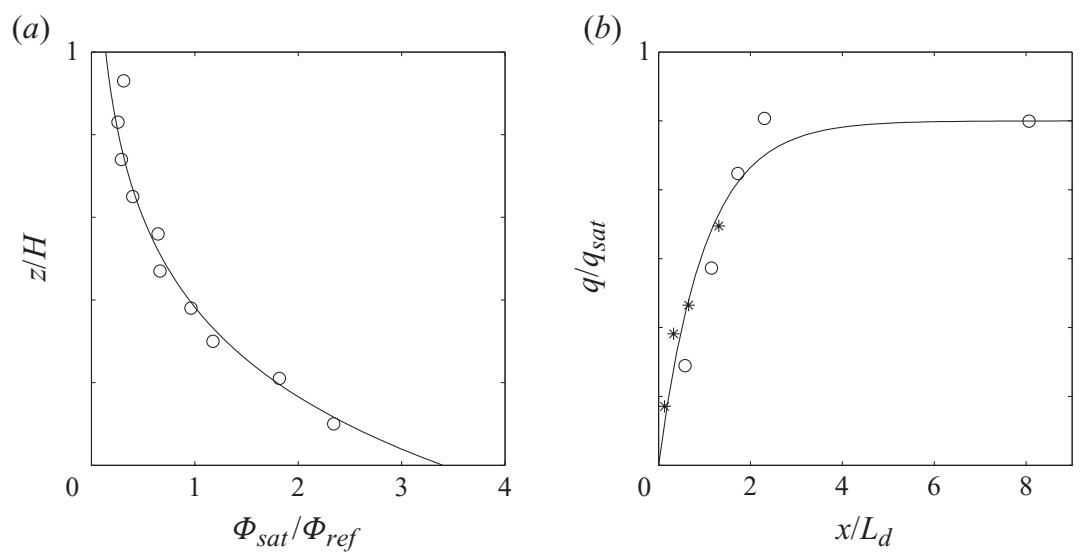

Figure 6. Net erosion experiments. (a) Concentration profile at the farthest location $x / L_{d}=8.1$, measured by Ashida \& Okabe (1982, run 5), normalized with the depth-averaged concentration $\Phi_{r e f}$ : solid line, $\Phi_{\text {sat }}$ given by (2.12) with $\alpha=3.2$. (b) Relaxation to saturation of the normalized sediment flux versus $x / L_{d}$ : symbols, van Rijn (1986a) and Ashida \& Okabe (1982) (see table 2); solid line, exponential relaxation (4.3).

The spatial evolution of the concentration profiles has been measured at different locations downstream of the transition point at $x=0$. The corresponding sediment flux $q$, which is zero for $x<0$, increases downstream until it reaches the saturated value $q_{\text {sat }}$. We determined this flux from integration of the measured concentration profile at each $x$-location. From the hydraulic parameters, the deposition length can be computed as $L_{d}=\left(U / V_{\text {fall }}\right) H$ - in the following we will not distinguish between $L_{1}$ and $L_{d}$, although they can differ by $\approx 20 \%$ for $\alpha$ of the order of unity (figure $3 b$ ). It appeared that for Ashida \& Okabe (1982) the location of the farthest downstream measurements corresponds to $x / L_{d}=8.1$, which is large enough for the sediment flux to be saturated. Figure $6(a)$ displays the corresponding concentration profile, and an exponential fit providing, from (2.12), the value of the parameter $\alpha$ reported in table 2 . For van Rijn (1986a), we found $x / L_{d}=1.3$, not large, preventing any straightforward determination of the parameter $\alpha$. For both experiments, the saturated flux $q_{\text {sat }}$ was estimated as that providing the best fit to the exponential curve,

$$
\frac{q}{q_{s a t}}=1-\exp \left(-x / L_{d}\right) \text {. }
$$

Figure $6(b)$ displays the variation of $q / q_{\text {sat }}$ with $x / L_{d}$; it can be seen that the data points fall quite well on the exponential curve. Note that the deposition lengths $L_{d}$ differ by one order of magnitude between the two experiments. The data collapse therefore supports a first-order relaxation process with characteristic length equal to $L_{d}$.

\subsection{Net deposition experiments}

Ashida \& Okabe (1982) have also performed experiments in which the initial concentration profile is over-saturated (run 6); i.e. the initial sediment flux $q_{0}$ at $x=0$ is larger than $q_{\text {sat }}$, so that the sediment settles until the saturated regime is reached further downstream. Figure $7(b)$ displays the sediment flux, obtained from the measured concentration profiles, together with the exponential relaxation curve 

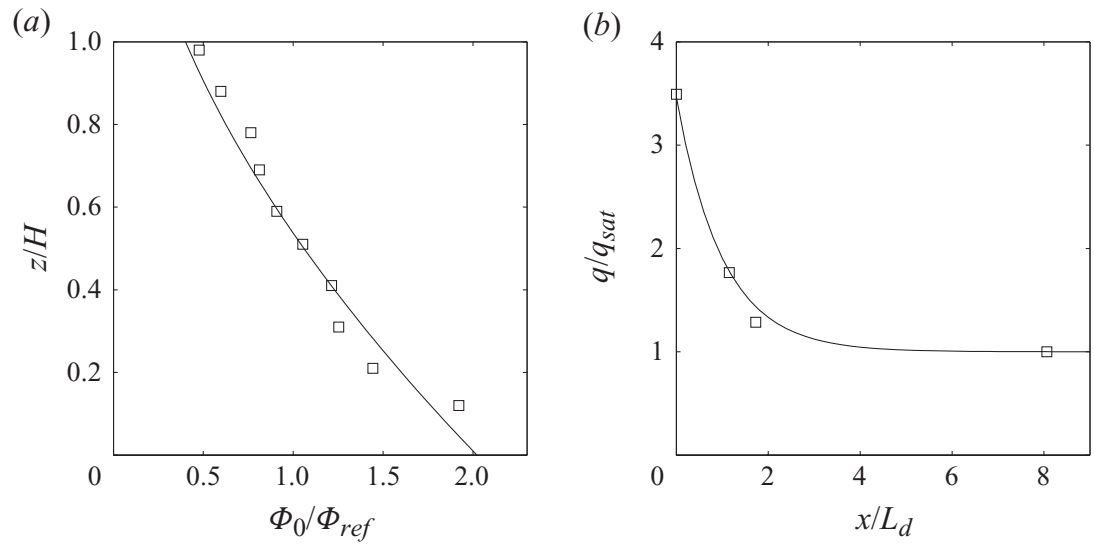

Figure 7. Net deposition experiments of Ashida \& Okabe (1982) (run 6; see table 2). (a) Concentration profiles at $x=0$ normalized with the depth-averaged concentration $\Phi_{\text {ref }}$ : solid line, $\Phi_{0}$ reconstructed from $\Phi_{\text {sat }}$ and one single mode for the concentration defect. $(b)$ Normalized sediment flux versus $x / L_{d}$, experiments and exponential relaxation (4.4).

now given by

$$
\frac{q}{q_{\text {sat }}}=1+\left(\frac{q_{0}}{q_{\text {sat }}}-1\right) \exp \left(-x / L_{d}\right),
$$

where $q_{0}$ and $q_{\text {sat }}$ were determined by curve fitting and $L_{d}$ is given in table 2 . Again, the agreement is quite good, showing that the mode with relaxation length $L_{d}$ captures most of the deposition process.

As a confirmation, figure $7(a)$ compares the concentration profile measured at the location $x=0$ with its projection over one single mode. This projection is computed from an empirical representation of this initial profile, using the expansion (3.12) and the saturated flux $\Phi_{\text {sat }}(z)$ measured from the concentration profile at $x=8.1 L_{d}$, fitted by the exponential form (2.12) with $\alpha=2.5$. It can be seen that the resulting profile is in good agreement with the measurements. Note that $\alpha=2.5$ corresponds to the Schmidt number $S c=\alpha \kappa u_{*} / 6 V_{\text {fall }}=0.33$, which is slightly below the usual range $0.5-1$ (Coleman 1970; Celik \& Rodi 1988; Nielsen 1992).

Other net deposition experiments have been performed by Jobson \& Sayre (1970). In this work, the particles were released near the water surface, so that the initial concentration profiles exhibit a peak close to $z=H$, as shown in figure $8(a)$. In contrast to the experiments of Ashida \& Okabe (1982), expanding the concentration defect over one single mode is not sufficient to get a good representation of this profile; an expansion over four modes provide a much better description, as shown in figure $8(a)$. However, the high-order modes are expected to vanish over a short distance, of the order of a few flow depths $H$, and the exponential relaxation to be recovered at large distances. This scenario is evidenced in figure $8(b)$, which displays the normalized flux versus $x / H$, measurements and the exponential curve (4.4). Here, owing to uncertainties in the falling velocity (see table 2 of the present paper and figure $6 a$ of Jobson \& Sayre 1970), the deposition length $L_{d}$ was determined, together with $q_{0}$ and $q_{\text {sat }}$, by fitting the experimental data points. We found $L_{d}=5.2 \mathrm{~m}$, which is close to the range of the expected values displayed in table 2, although slightly smaller. We finally note that in the course of the reconstruction of $\Phi_{0}(z)$, we found 
(a)

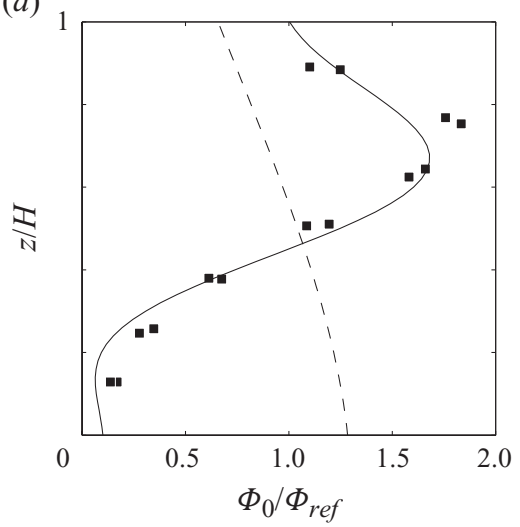

(b)

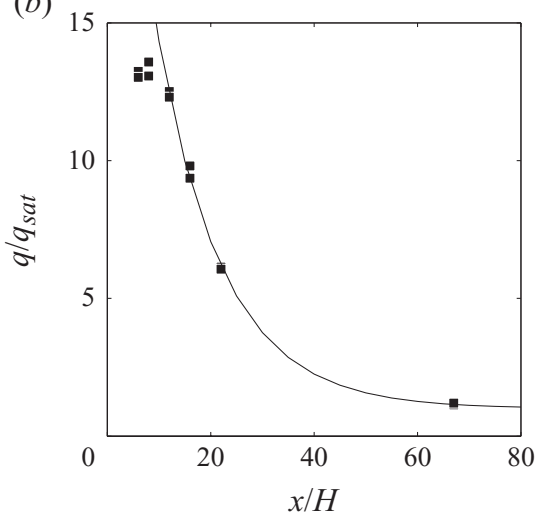

Figure 8. Net deposition experiments of Jobson \& Sayre (1970) (see table 2). (a) Measured concentration profile at $x=0$ normalized with the depth-averaged concentration $\Phi_{\text {ref }}$ : dashed and solid lines, $\Phi_{0}$ reconstructed with one single mode and four modes, respectively, and $\alpha=1.2$. (b) Relaxation to saturation of the normalized sediment flux versus $x / L_{d}$ : solid line, exponential relaxation (4.4) with $L_{d}=5.2 \mathrm{~m}$.

$\alpha=1.2$ from the saturated concentration profile, which corresponds to the Schmidt number in the range $0.18-0.36$, slightly smaller, again, than the usual range.

\section{Concluding remarks}

In this paper, we have discussed the conditions under which a first-order relaxation equation for the sediment flux $q$ can be derived for turbulent flows, when suspension is the dominant mode of transport. From a mode analysis of the linear advectiondiffusion equation for the particle concentration, it was shown that the sediment flux is dominated by the mode corresponding to the largest relaxation length for spatially varying flows, or the largest relaxation time for time-dependent flows. These relaxation scales were identified as the deposition length $H U / V_{\text {fall }}$ and the deposition time $H / V_{\text {fall }}$, where $H$ is the flow depth, $U$ the mean flow velocity and $V_{\text {fall }}$ the sediment settling velocity. This result is expected to be particularly relevant for the case of sediment transport in slowly varying flows, for which the flux is never far from saturation. Predictions of the sediment flux were shown to be in quantitative agreement with flume experiments, for both net erosion and net deposition situations, and deposition lengths spanning over one order of magnitude.

As discussed in the Introduction, the relaxation equation (1.1) allows for the description of both bed and suspended loads. However, these modes of transport correspond to very different physical length scales. For bed load, the relaxation length $L_{\text {sat }}$ is of the order of 10 grain diameters (Fourrière et al. 2010). As soon as the flow depth $H$ is larger than a few $L_{\text {sat }}$, the 'unstable' flat bed is insensitive to the presence of the free surface, and current ripples emerge at a centimetric wavelength $\left(\approx 10 L_{\text {sat }}\right)$. When suspended load is the dominant type of transport, we have shown that the relaxation length $L_{\text {sat }}$ is of the order of 10-100 $\mathrm{H}$, which is typically four to five orders of magnitude larger than for bed load. Suspended transport thus prevents the formation of bedforms with a wavelength smaller than $H$, and patterns such as bars, anti-dunes and meanders can be expected to emerge from linear instability, with large wavelengths of the order of 100-1000 H. Further work is required for the experimental and theoretical investigations of these instabilities. 
This work has benefited from the financial support of the Agence Nationale de la Recherche, grant Zephyr (no. ERCS07_18) and the GdR MéPhy of the CNRS (no. 3166).

\section{REFERENCES}

Andreotti, B. 2004 A two species model of aeolian sand transport. J. Fluid Mech. 510, 47-50.

Andreotti, B., Claudin, P. \& Douady, S. 2002 Selection of dune shapes and velocities. Eur. J. Phys. B 28, 341.

Apmann, R. P. \& Rumer, R. R. 1970 Diffusion of sediment in developing flow. J. Hydraul. Div. ASCE 96, 109-123.

AsHidA, K. \& OKABE, T. 1982 On the calculation method of the concentration of suspended sediment under non-equilibrium condition (in Japanese). In Proceedings of the 26th Conference on Hydraulics, pp. 153-158. JSCE.

Bec, J., Biferale, L., Cencini, M., Lanotte, A., Musacchio, S. \& Toschi, F. 2007 Heavy particle concentration in turbulence at dissipative and inertial scales. Phys. Rev. Lett. 98, 084502.

Bonelli, S., Brivois, O. \& BenAhmed, N. 2007 Modélisation du renard hydraulique et interprétation de l'essai d'érosion de trou. Rev. Fr. Geotech. 118, 13-22.

Briaud, J. L., Ting, F. C. K., Chen, H. C., CaO, Y., Han, S. W. \& Kwak, K. W. 2001 Erosion function apparatus for scour rate predictions. J. Geotech. Geoenviron. Engng 127, 105-113.

CELIK, I. \& RoDI, W. 1988 Modeling suspended sediment transport in nonequilibrium situations. J. Hydraul. Engng 114, 1157-1191.

Charru, F. 2006 Selection of the ripple length on a granular bed sheared by a liquid flow. Phys. Fluids 18, 121508.

Coleman, N. L. 1970 Flume studies of the sediment transfer coefficient. Water Resour. Res. 6, 801-809.

Davy, P. \& Lague, D. 2009 Fluvial erosion/transport equation of landscape evolution models revisited. J. Geophys. Res. 114, F03007.

Einstein, H. A. 1950 The bedload function for sediment transportation in open channel flow. Tech. Bull. 1026, pp. 1-71. US Department of Agriculture.

Engelund, F. 1970 Instability of erodible beds. J. Fluid Mech. 42, 225-244.

Fourrière, A., Claudin, P. \& Andreotti, B. 2010 Bedforms in a turbulent stream: formation of ripples by primary linear instability and of dunes by non-linear pattern coarsening. J. Fluid Mech. 649, 287-328.

Fredsøe, J. \& DeigaArd, R. 1992 Mechanics of Coastal Sediment Transport. World Scientific.

Hanson, G. J. \& Simon, A. 2001 Erodibility of cohesive streambeds in the loess area of the midwestern USA. Hydraul. Process. 15, 23-38.

Huelmfelt, A. T. \& Lenau, C. W. 1970 Nonequilibrium transport of suspended sediment. J. Hydraul. Div. ASCE 96, 1567-1586.

Hunt, J. C. R., Delfos, R., Eames, I. \& Perkins, R. 2007 Vortices, complex flows and inertial particles. Flow Turbul. Combust. 79, 207-234.

Jobson, A. E. \& SAYre, W. W. 1970 Vertical transfer in open channel flow. J. Hydraul. Div. ASCE 96, 703-724.

Kroy, K., Sauermann, G. \& Herrmann, H. J. 2002 Minimal model for aeolian sand dunes. Phys. Rev. E 66, 031302.

Lajeunesse, E., Malverti, L. \& Charru, F. 2010 Bedload transport in turbulent flow at the grain scale: experiments and modeling. J. Geophys. Res. 115, F04001.

MeI, C. C. 1969 Nonuniform diffusion of suspended sediment. J. Hydraul. Div. ASCE 95, 581-584.

NezU, I. 2005 Open-channel flow turbulence and its research prospect in the 21st century. J. Hydraul. Engng 131, 229-246.

Nezu, I. \& Rodi, W. 1986, Open-channel flow measurements with a laser doppler anemometer. J. Hydraul. Engng. 112, 335-355.

Nielsen, P. 1992 Coastal Bottom Boundary Layers and Sediment Transport. World Scientific.

Ouillon, S. \& Le GuenneC, B. 1996 Modelling non-cohesive suspended sediment transport in 2D vertical free surface flows. J. Hydraul. Res. 34, 219-236. 
PARKER, G. 1978 Self-formed straight rivers with equilibrium banks and mobile bed. Part 1. The sand-silt river. J. Fluid Mech. 89, 109-125.

RaudKivi, A. J. 1998 Loose Boundary Hydraulics. A. A. Balkema.

VAN RIJN, L. C. $1984 a$ Sediment pick-up functions. J. Hydraul. Engng 110, 1494-1502.

VAN RiJn, L. C. $1984 b$ Sediment transport. Part II. Suspended load transport. J. Hydraul. Engng 110, 1613-1641.

VAN RIJN, L. C. $1986 a$ Application of sediment pickup function. J. Hydraul. Engng 112, 867-874.

VAN RIJN, L. C. $1986 b$ Mathematical modeling of suspended sediment in nonuniform flows. J. Hydraul. Engng 112, 433-455.

Rouse, H. 1936 Modern conceptions of the mechanics of fluid turbulence. Trans. ASCE 102, $463-543$.

Sauermann, G., Kroy, K. \& Herrmann, H. J. 2001 A phenomenological dynamic saltation model for dune formation. Phys. Rev. E 64, 031305.

Seminara, G. 2006 Meanders. J. Fluid Mech. 554, 271-297.

SHIELDS, A. 1936 Application of similarity principles and turbulence research to bed-load movement. Mitteilungen der Preussischen Versuchsanstalt für Wasserbau und Schiffbau (ed. W.D. Ott \& C. von Uchelen), vol. 26, pp. 5-24. Soil Conservation Service, California Institute of Technology.

VANONI, V. A. 1946 Transportation of suspended sediment by water. Trans. ASCE 111, 67-133.

Yalin, K. S. \& Finlaysen, G. D. 1973 On the development of the distribution of suspended load. In Proceedings of the 15th IAHR Congress, Istanbul (ed. S. Okyay), pp. 287-294. 